\title{
An explorer and surveyor
}

\author{
Hermann Weyl made prescient contributions to both mathematics and physics, but also strove to \\ understand reality as a whole.
}

\section{Frank Wilczek}

Hermann Weyl was, according to Fields medallist Michael Atiyah, "one of the greatest mathematicians of the first half of the twentieth century". Every great mathematician is great in their own way, but Weyl's way was particularly special. Unlike most modern scientists who choose one or a few specific areas to explore and look neither sideways nor back, Weyl surveyed the whole world. He sought truth and beauty with a discriminating and far-seeing eye.

Weyl's most unique work is Philosophy of Mathematics and Natural Science. No one else could have written it, and no other book I know is like it. I have consulted it many times, and each time I've come away enriched. My main purpose here is to direct readers to that book. But since space remains, let me add some background information.

Weyl, who died in 1955 at the age of 70 , was a student of David Hilbert at the GeorgAugust University in Göttingen, Germany, and thus stood in the line of intellectual descent from Carl Gauss, Bernhard Riemann and Lejeune Dirichlet. Upon Hilbert's retirement, Weyl was invited to take up his chair, but conditions in 1930s Germany and an attractive offer from the new Institute for Advanced Study conspired to bring him to Princeton, where he stayed. With Albert Einstein and John von Neumann, Weyl made the trinity of refugee stars that gave the institute its inimitable scientific lustre.

Einstein and von Neumann had both grown up in the grand German literary and pan-European cultural tradition that was rocked and then shattered by the two world wars. But more than the rebellious Einstein or the protean von Neumann, Weyl embodied that tradition, and his Philosophy of Mathematics and Natural Science reflects it in focus, style and erudition.

The main body of text was written in German in 1926, as an article for R. Oldenburg's Handbuch der Philosophie. In 1949, for the English translation, Weyl altered many details and added seven appendices, comprising almost a hundred pages. These centre on relevant scientific events in the intervening years - his passages on Goddel's theorem, and on quantum mechanics and causality are especially brilliant. But the core of the book had its genesis in the vanished handbook tradition of magisterial

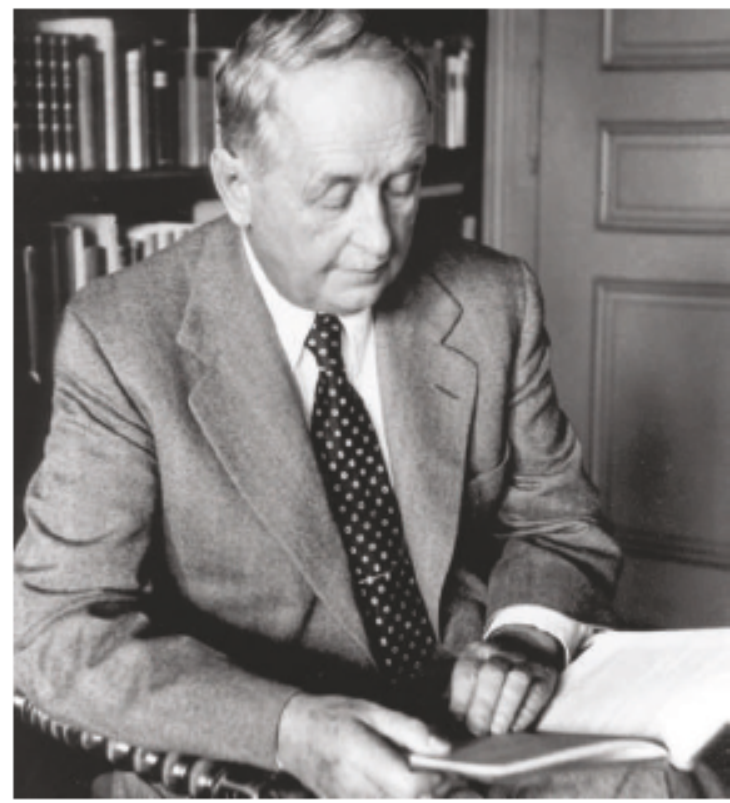

Hermann Weyl touched on questions that are relevant today.

reviews in natural philosophy.

In the introduction, Weyl declares the roots of his literary style: "I was bound by the German literary and philosophical tradition in which I grew up." As an example of that style, let me quote a passage that I think ranks among the most beautiful and profound passages in all literature: ${ }^{\alpha}$ The objective world simply is, it does not happen. Only to the gaze of my consciousness, crawling along the lifeline of mybody, does a section of this world come to life as a fleeting image in space which continuously changes in time."

Weyl's work is also remarkable for its erudition. René Descartes, Gottfried Leibniz, David Hume and Immanuel Kant enter into the discussion as familiar friends. From time to time important literary or philosophical passages are quoted in the original French, German, or even Greek. Weyl's erudition is not vain display - he is far above that - but a touching assumption of shared culture. Of course the reader will feel at home in this milieu, he seems to assume.

In his biographical memoir of Weyl, Atiyah also talked about Weyl's work in mathematics, saying: "The last 50 years have seen a remarkable blossoming of just those areas that Weyl initiated. In retrospect, one might almost say that Weyl defined the agenda and provided the proper framework for what followed. Weyl'scontributions in physics were more sporadic, but they were significant, $\frac{}{6}$ and likewise remarkably है prescient. In particular, Weyl proposed, and named, the concept of gauge invariance', which came to dominate fundamental physics during the second half of the twentieth century.

Evidently, Weyl's intuitions have an excellent \% track record. By now, parts of the Philosophy of Mathematics and Natural Science are dated, of course. Those parts retain interest as intellectual history, because Weyl's understandings, being close to the best that was possible in their time, serve as benchmarks. But beyond that, Philosophy of Mathematics and Natural Science touches great questions that remain very much alive. Near the end of the book, after a penetrating critique of the concept of causality, Weyl turns to what he calls the body-soul problem - what we know today as the problem of consciousness.

${ }^{\alpha}$ It is an altogether too mechanical conception of causality that views the mutual effects of body and soul as being so paradoxical that one would rather resort, like Descartes, to the occasionalistic intervention of God or, like Leibniz, to a harmony instituted at the beginning of time.

The real riddle, if I am not mistaken, lies in the double position of the ego: it is not merely an existing individual which carries out real psychic acts, but also 'vision, a selfpenetrating light (sense-giving consciousness, knowledge, image, or however you may call it); as an individual capable of positing reality, its vision open to reason; 'a force into which an eye has been put,' as Fichte says."

The fullest enlightenment comes only to those who, like Weyl, discern its absence, seek it, and recognize it when it arrives. Frank Wilczek is at the Center for Theoretical Physics, Massachusetts Institute of Technology, Cambridge, Massachusetts, 02142, USA.

\section{FURTHER READING}

Atiyah, M. in BiographicalMemoirs Vol. 82,1-1B (Natl Acad, Washington DC, 2002).

Weyl, H. Ahilosaphy of Mathematics and Natural

Science (PrincetonUniv. Press, Princeton, 1949). 